\title{
Deep Sequencing Identified Dysregulated Circulating MicroRNAs in Late Onset Preeclampsia
}

\author{
DANAI MAVRELI ${ }^{1,2^{*}}$, ALEXANDRA LYKOUDI ${ }^{1,2^{*}}$, GEORGE LAMBROU ${ }^{3}$, GEORGE PAPAIOANNOU $^{1}$, \\ NIKOLAS VRACHNIS ${ }^{1}$, SOPHIA KALANTARIDOU ${ }^{1}$, NIKOLAS PAPANTONIOU ${ }^{1}$ and AGGELIKI KOLIALEXI ${ }^{1,2}$ \\ ${ }^{1} 3^{\text {rd }}$ Department of Obstetrics and Gynecology, National and Kapodistrian University of Athens, Athens, Greece; \\ ${ }^{2}$ Department of Medical Genetics, National and Kapodistrian University of Athens, Athens, Greece; \\ ${ }^{3} 1^{\text {st }}$ Department of Pediatrics, National and Kapodistrian University of Athens, Athens, Greece
}

\begin{abstract}
Background/Aim: To characterize global microRNA (miRNA) expression profile in the first trimester maternal plasma of women who subsequently develop lateonset preeclampsia (LOPE) compared to uncomplicated pregnancies. Materials and Methods: Five first trimester plasma samples from women who developed LOPE and 5 controls were analyzed using next generation sequencing technology (NGS) followed by target prediction, Gene Ontology analysis and pathway identification. Quantitative real-time polymerase chain reaction ( $q R T-P C R)$ was performed for confirmation in an independent cohort of 12 LOPE cases and 12 controls. Results: miR-23b-5p and miR99b-5p were down-regulated by $>1.5$ fold in LOPE complicated pregnancies ( $p$ value $<0.05)$ compared to controls. Target prediction showed that the major targets of these miRNAs are associated with glycometabolism and immune response. Conclusion: $m i R-23 b-5 p$ and miR-99b-5p are possibly implicated in the pathogenic mechanisms leading to the induction of LOPE and may serve as candidate noninvasive biomarkers for early prediction and prevention.
\end{abstract}

Preeclampsia (PE) (1), a multisystem pregnancy complication affecting $2-8 \%$ of all pregnancies globally, is one of the major contributors to maternal and fetal mortality and morbidity accounting for $10-15 \%$ of maternal deaths (1). It is clinically defined as pregnancy-induced hypertension $(>140 / 90 \mathrm{mmHg}$

This article is freely accessible online.

*These Authors contributed equally to this work.

Correspondence to: Aggeliki Kolialexi, Department of Medical Genetics, Athens University School of Medicine, Thivon and Levadias, 11527 Athens, Greece. Tel: +30 2107467462, Fax: +30 2107795553, e-mail: akolial@med.uoa.gr

Key Words: microRNAs, NGS, preeclampsia, late-onset preeclampsia, miR- 23b-5p, miR-99b-5p. on two separate occasions) together with proteinuria $(>300$ $\mathrm{mg} /$ day). In the absence of significant proteinuria, hypertension in the presence of features associated with maternal endothelial dysfunction such as thrombocytopenia, renal insufficiency, impaired liver function, pulmonary edema, cerebral or visual symptoms, during the second or third trimester of pregnancy is sufficient to support a diagnosis (2).

Although the disease typically resolves after delivery, women who experience PE and their neonates are at increased risk for cardiovascular and metabolic disease in later life $(3,4)$. Setting the cut-off point at 34 weeks gestation, PE is classified either as early onset (EOPE) which develops before 34 weeks of gestation, or the more common late-onset sybtype (LOPE), which develops at or after 34 weeks of gestation $(4,5)$. The two subtypes have different etiology and develop through different pathophysiological mechanisms. Poor placentation and impaired spiral artery remodeling are linked with EOPE, whereas LOPE is possibly the result of a mismatch between the metabolic demands of the growing fetus and maternal supplies.

Induced preterm delivery remains the only treatment option for managing PE. Nevertheless, recent evidence suggests that early prediction of women at risk for PE may either prevent disease onset or minimalize adverse pregnancy outcome with the administration of Aspirin ${ }^{\circledR}$ before the $16^{\text {th }}$ week of pregnancy (6). Several risk factors from maternal characteristics, obstetrics, and medical history, including ethnicity, age, parity, multiple pregnancy, and a history of PE in earlier pregnancy, combined with biophysical markers [uterine artery pulsatility index (UtA-PI)] and biochemical markers such as soluble fms-like tyrosine kinase 1 (sFlt1), pregnancy associated plasma protein-A (PAPP-A), vascular endothelial growth factor (VEGF) and placental growth factor (PlGF) have been investigated for their ability to predict PE at 11-13 weeks of gestation. So far, an algorithm, which combines maternal factors, mean arterial pressure, UtA-PI and maternal serum PIGF at 11-13 weeks' gestation, has achieved the best performance. The estimated detection 
rate (DR) of screening using this model is $75 \%(95 \%$ confidence interval $=70-80 \%)$ for EOPE and $47 \%(95 \%$ confidence interval $=44-51 \%$ ) for LOPE at $10 \%$ false positive rate (FPR) (7). Thus, there is a real clinical need to identify biomarkers with predictive value in particular for LOPE.

MicroRNAs (miRNAs), a class of small non-coding endogenous RNA molecules of $\sim 21-24$ nucleotides that transcriptionally regulate gene expression, have attracted the attention of researchers due to their crucial role in several biological processes including cell growth, proliferation, differentiation and apoptosis (8-11).

MiRNAs are expressed within cells as well as in extracellular fluids such as serum, plasma, saliva, urine and milk. These extracellular miRNAs known as circulating miRNAs are characterized by increased stability, up to $10 \times$ higher than that of messenger RNA (12). Due to their noninvasive nature, stability and ease of quantitation, circulating miRNAs have been considered as promising biomarkers for various pathologic conditions including cancer, cardiovascular diseases and pregnancy complications (13). Recent evidence suggest that miRNAs are implicated in the regulation of placentation, angiogenesis, blood pressure and inflammatory response highlighting their involvement in the pathogenesis of the disease as well as an important role as candidate biomarkers for the early prediction of PE.

In the present study, miRNA profiling of the first trimester maternal plasma from women who subsequently developed LOPE along with healthy controls was performed, using Next Generation Sequencing technology followed by quantitative real-time polymerase chain reaction (qRT- PCR) verification, in order to identify candidate biomarkers for the early prediction of the complication and suggest pathogenic mechanisms associated with the development of PE. The analysis focused on LOPE, which accounts for approximately $85 \%$ of PE complicated pregnancies.

\section{Materials and Methods}

Study population. For this retrospective case-control study maternal peripheral blood samples were obtained from a non-selected population of 2437 Caucasian pregnant women at $11^{+0}$ to $13^{+6}$ weeks of gestation undergoing first trimester prenatal screening for fetal aneuploidies using ultrasonographic and biochemical markers. During this first visit, blood samples were collected using Ethylenediamine-tetra acetic acid (EDTA) Vacutainer tubes (Becton Dickinson UK Ltd, Oxfordshire, United Kingdom) and processed within $2 \mathrm{~h}$ from collection by double centrifugation at $2500 \times \mathrm{g}$ for $10 \mathrm{~min}$ at $4^{\circ} \mathrm{C}$ for plasma separation. Plasma was retained into 250 $\mu 1$ RNA/DNA enzyme-free frozen eppendorfs (ThermoFisher Scientific, Waltham, MA, USA) and immediately stored at $-80^{\circ} \mathrm{C}$ until RNA extraction.

The demographic and clinical data of all women participating in the study were collected, using a pre-established questionnaire, by the scientists involved in the project and recorded in an electronic database. In all cases gestational age was calculated based on the
Table I. General characteristics of the study population.

\begin{tabular}{lccc}
\hline & $\begin{array}{c}\text { LOPE* } \\
(\mathrm{n}=17)\end{array}$ & $\begin{array}{c}\text { Controls } \\
(\mathrm{n}=17)\end{array}$ & \\
& Median (Min, Max) & Median (Min, Max) & $p$-Value \\
\hline $\begin{array}{l}\text { Gestational age } \\
\text { at blood collection } \\
\text { (weeks) }\end{array}$ & $12.43(11.80,14.14)$ & $12.29(11.43,13.00)$ & 0.17 \\
$\begin{array}{l}\text { Maternal age } \\
\text { (MA) (years) }\end{array}$ & $31.81(21.28,39.50)$ & $33.19(26.75,41.27)$ & 0.09 \\
$\begin{array}{l}\text { Body-mass index } \\
\text { (BMI) }\end{array}$ & $27.10(18.60,46.90)$ & $22.60(19.10,44.80)$ & 0.30 \\
$\begin{array}{c}\text { Smoking } \\
\text { Yes }\end{array}$ & $3(17.6 \%)$ & $6(35.2 \%)$ & - \\
$\begin{array}{c}\text { No } \\
\text { Gestational age at } \\
\text { delivery (Years) }\end{array}$ & $36.40(34.40,38.50)$ & $39.80(39.00,40.50)$ & 0.00 \\
$\begin{array}{l}\text { Fetal birth weight } \\
\text { (BW) (gr) }\end{array}$ & 2860.00 & 3445.00 & 0.50 \\
Fetal gender & $(2410.00,3280.00)$ & $(2950.00,30500.00)$ & \\
$\begin{array}{l}\text { Boys } \\
\text { Girls }\end{array}$ & $10(59 \%)$ & $9(53 \%)$ & - \\
\hline
\end{tabular}

crown-rump length (CRL) at first-trimester ultrasound (14). Following delivery, information on pregnancy outcome was available from the hospital records. Based on these data, 34 samples were retrieved for analysis, comprising 17 from women who subsequently developed LOPE and required delivery at $>34$ weeks of gestation and 17 from uncomplicated pregnancies (controls) matching for maternal age, gestational age and duration of storage of plasma samples. Patient demographic characteristics and clinical data are presented in Table I. Subsequently, samples were randomly divided into screening and verification sets. During the first phase of the study, the biomarker-screening phase, samples from 5 women who later developed LOPE and 5 controls were used for miRNA profiling using the NGS technology for the identification of circulating miRNAs showing significant difference in expression levels between the LOPE and control samples. In the second phase, the verification step, the differential expression of previously identified miRNAs was confirmed by qRT-PCR in an independent group of 12 samples obtained from women with subsequent LOPE and 12 from uncomplicated pregnancies.

Women included in the study had a singleton pregnancy, delivered phenotypically normal neonates and, at the time of blood sampling, were normotensive and did not receive any medication. None of the samples were previously thawed and refrozen.

PE was defined as new-onset hypertension that developed after 20 weeks of gestation (systolic or diastolic blood pressure $\geq 140$ and/or $\geq 90 \mathrm{~mm} \mathrm{Hg}$, respectively, measured on at least two occasions, $4 \mathrm{~h}$ to 1 week apart) and proteinuria ( $\geq 300 \mathrm{mg}$ in a $24-\mathrm{h}$ urine collection, or two random urine specimens obtained $4 \mathrm{~h}$ to 1 week apart containing $\geq 1+$ by dipstick) (15).

LOPE was defined as preeclampsia diagnosed at or after 34 weeks of gestation.

Normal pregnancies were defined as those without complications that delivered at $38 \pm 42$ weeks of gestation a chromosomally normal baby weighting within the normal range for gestational age. 

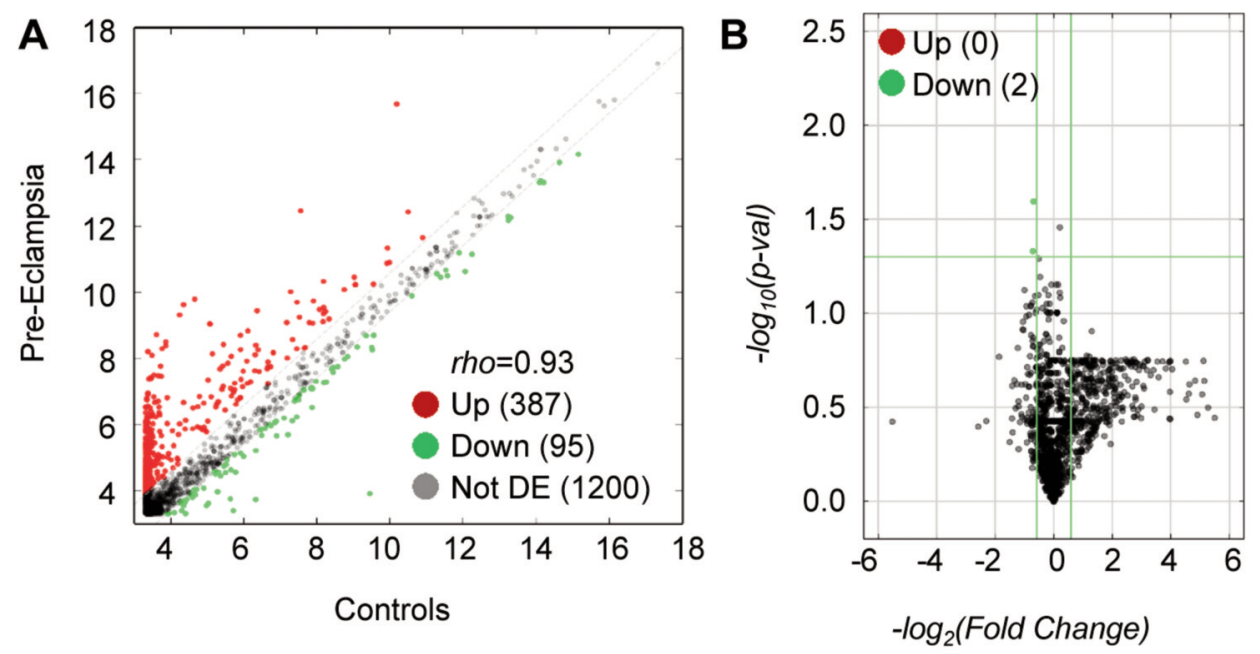

Figure 1. Expression profile of miRNAs in the first trimester maternal plasma in women who subsequently developed LOPE and uncomplicated pregnancies: A. Scatter plot comparing the miRNA expression profile in LOPE cases and controls. B. Volcano plot. The green dots in the plot represent the differentially expressed miRNAs with statistical significance (Fold Change $>1.5$ and $p<0.05$ ).

Circulating miRNAs extraction. Plasma samples were thawed on ice and centrifuged at $3.000 \times \mathrm{g}$ for $5 \mathrm{~min}$ in order to remove cellular debris that may affect the miRNA profiling. Isolation of plasma total RNA (including miRNAs) was carried out using the miRCURY RNA isolation kit (Exiqon, Vedbaek, Denmark) following the manufacturer's protocol. RNA concentration was estimated by OD260/OD280 ratio on a NanoDrop ND-1000 spectrophotometer (Thermo Fisher Scientific). The RNA integrity was examined with an Agilent Bioanalyzer2100 (Agilent, Santa Clara, CA, USA).

Small RNA library preparation and NGS analysis. Total RNA from each case was used to construct small RNA libraries using the Illumina TruSeq Small RNA library kit (Illumina, San Diego, CA, USA). Briefly, total RNA of each sample was sequentially ligated to 3'and 5' small RNA adapters, reverse transcribed and amplified using Illumina's proprietary amplification primers. Subsequently, amplified fragments of $130-150 \mathrm{bp}$, corresponding to $\sim 15-35 \mathrm{nt}$ small RNAs, were size selected using $6 \%$ polyacrylamide gel electrophoresis and the concentration of each library was determined using an Agilent 2100 Bioanalyzer (Agilent). The DNA fragments in the libraries were denatured with $0.1 \mathrm{M} \mathrm{NaOH}$ to generate single-stranded DNA molecules, captured on Illumina flow cells, amplified in situ and finally sequenced in 51 cycles on Illumina Nextseq 500 (Illumina, San Diego, CA, USA) highthroughput sequencing according to the manufacturer's instructions.

Raw data processing and miRNAs differential expression. Raw sequences were generated as clean reads from Illumina Next seq 500 by real-time base calling and quality filtering. The 3' prime adapter sequences were trimmed and reads with lengths $<15$ nucleotides were discarded. The trimmed reads were recorded in FASTA format and those with length ${ }^{3} 15 \mathrm{nt}$ were aligned to the human pre-miRNA database (miRBase v21) using NovoAlign software (NovoCraft, Selangor, Malaysia) allowing maximum one mismatch per sequence. Reads with counts $<2$ were discarded when calculating the miRNA expression. The alignment results were saved in text files. The miRNA expression levels were measured and normalized as transcripts per million (TPM) of total aligned miRNA reads (16). The miRNA read counts are used to estimate the expression levels of each miRNA. The sequence counts for all miRNAs identified were compiled in an Excel file.

For the identification of differentially expressed miRNAs in LOPE cases as compared to uncomplicated pregnancies, the fold change (FC) and $p$-values were computed. The Students $t$-test was used for differential expression analysis. MiRNAs having $\mathrm{FC} \geq 1.5$ and $p$-value $\leq 0.05$ were considered significant. miRNA relative expression was estimated as the $\log 2$-transformed ratio of an individual miRNA expression level over the mean expression level of all control samples for the respective miRNA under investigation.

Quantitative real-time polymerase chain reaction ( $q R T-P C R)$. The differential expression of miR-99b-5p and miR-23b-5p in LOPE complicated pregnancies, as compared to the control group, was verified using qRT-PCR. Complementary DNA (cDNA) synthesis and subsequent qRT-PCR were performed using the TaqMan miRNA Reverse Transcription kit and TaqMan universal PCR master mix (Applied Biosystems, Foster City, CA, USA) respectively. Specific primers and a TaqMan probe for miR-99b-5p and miR-23b-5p (Applied Biosystems, Inc.) were used according to the manufacturer's instructions. All samples were analyzed in triplicate in an LC480 LightCycler system (Roche $\mathrm{GmbH}$, Basel, Switzerland). Positive amplification of each sample was considered when a signal occurred before the 40th threshold cycle. The miRNA levels were normalized to U6 (Applied Biosystems, Inc.) as an internal control and the relative abundance of each miRNA was calculated using the $\Delta \Delta C t$ equation.

Statement of ethics. The study was performed according to the Helsinki Declaration on ethical principles for medical research involving human subjects. All samples were obtained after a written informed consent was received and the ethics committee at 


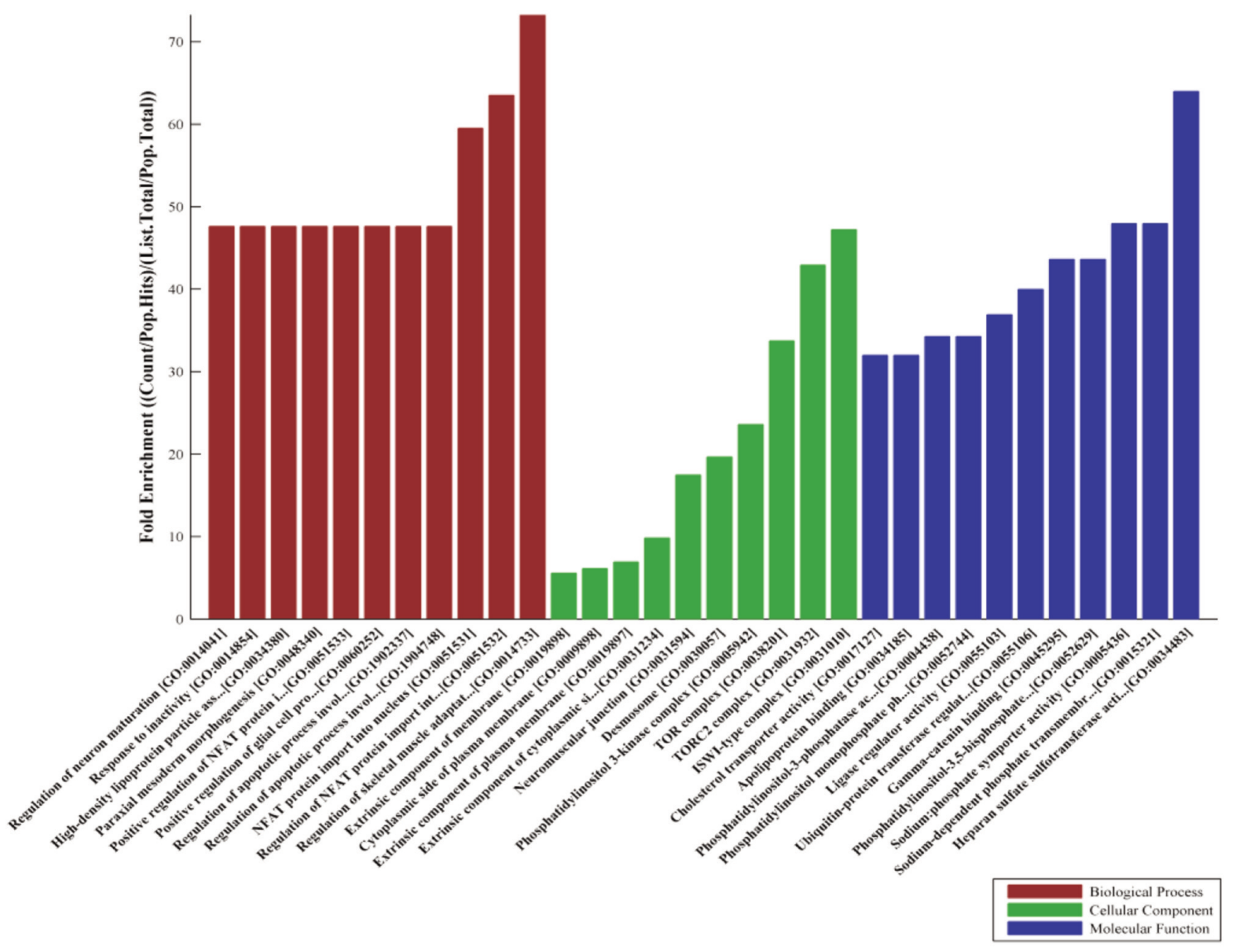

Figure 2. Gene ontology $(G O)$ categories enriched with predicted targets of miRNAs differentiating LOPE complicated pregnancies from controls.

"Attikon" University Hospital approved the study protocol (approval reference number: 27-268 ex 14/15).

Data analysis and statistics. The MATLAB $^{\circledR}$ simulation environment (The Mathworks, Inc, Natick, MA, USA) was applied for statistical analysis. Differences in clinical characteristics between the two groups were assessed using one-way ANOVA for independent variables or the Mann-Whitney $U$-test for continuous variables. Receiver operating characteristic (ROC) curves were performed to evaluate the diagnostic potential of differentially expressed miRNAs with 95\% standard error (SE) and 95\% confidence intervals (CI).

miRNA enrichment, gene ontology and pathway analysis. Differentially expressed miRNAs were further enriched and analyzed for known functions using Webgestalt web-tool (17). The TargetScan, PicTar and miRanda algorithms were applied in combination to identify the potential target genes of differentially expressed miRNAs. In the gene ontology analysis (GO), the number of genes corresponding to GO entries namely Biological Process,
Molecular function and Cellular Component, were determined and the enrichment score was reported as the $-\log$ ( $p$-value). The data obtained were uploaded into the Database for Annotation, Visualization and Integrated Discovery (DAVID), version 6.7, a database with integrated biological information and analytical tools, for GO and pathway identification using the Kyoto Encyclopedia of Genes and Genomes (KEGG) database.

\section{Results}

MicroRNA Differential Expression Profiles between PE and the control group. A total of 1682 miRNAs were identified in the first trimester maternal plasma using an Illumina Next Generation Sequencing platform. Among these, 387 were up-regulated, 95 down-regulated and 1200 demonstrated similar expression levels between the two groups (Figure 1A). Statistical analysis revealed that two miRNAs, namely miR-99b-5p and miR-23b-5p, showed 


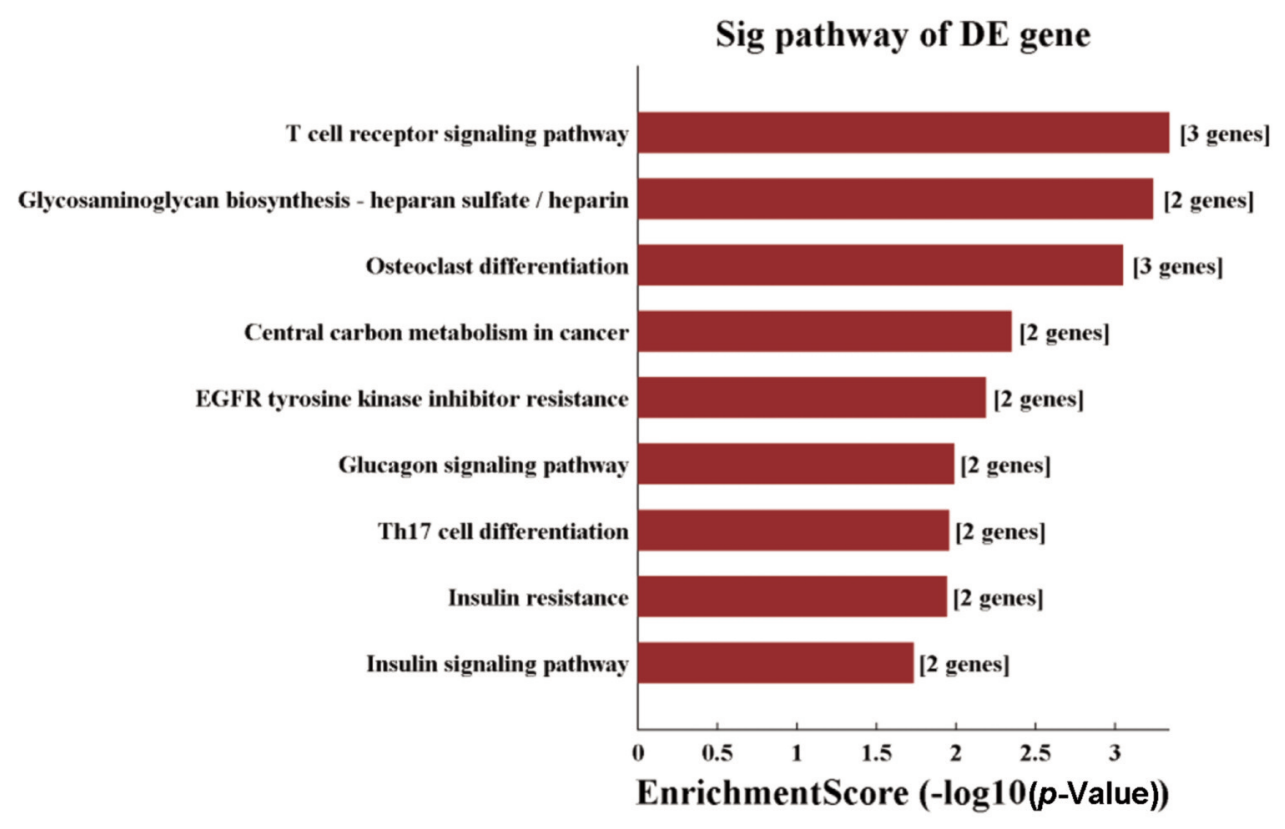

Figure 3. Kyoto Encyclopedia of Genes and Genomes pathways enriched with annotated differentially expressed miRNAs (miR-23b and miR99b).

significantly altered expression in the first trimester maternal plasma of women at risk for LOPE as compared to the control cohort. The significantly differentially expressed miRNAs are presented in a Volcano plot (Figure 1B). Both miRNAs were down-regulated; $\log 2$, $m i R-99 b-5 p=-0.69$ and $\log 2, m i R-23 b-5 p=-0.72$ with $p$ values 0.025 and 0.046 , respectively.

NGS data are available online on the Gene Expression Omnibus (GEO) under the GEO accession number GSE119799. Other raw data are available upon reasonable request.

miRNA enrichment, gene ontology and pathway analysis. A total of 188 putative target mRNAs of the two differentially expressed miRNAs were predicted using MiRanda, TargetScan and Pic T algorithms. In the GO analysis, the number of genes corresponding to GO entries was determined and the enrichment score was reported as the -log ( $p$-value). The most enriched GO terms for biological processes, molecular functions and cellular components are presented in Figure 2.

Pathway analysis resulted in a list of 9 pathways with significant enrichment for the predicted miRNA target genes (Figure 3). These pathways include: Th17 cell differentiation (has $04659, p=0.01$ ); $\mathrm{T}$ cell receptor signaling pathway (has 04660, $p=0.00046$ ); Osteoclast differentiation (has 04380, $p=0.00091$ ); Insulin signaling pathway (has 04910, $p=0.019$ ); Insulin resistance (has 04931, $p=0.011$ ); Glycosaminoglycan biosynthesis-heparan sulfate/heparin (has 00534, $p=0.0006$ ); Glucagon signaling pathway (has 04922, $p=0.011$ ); EGFR

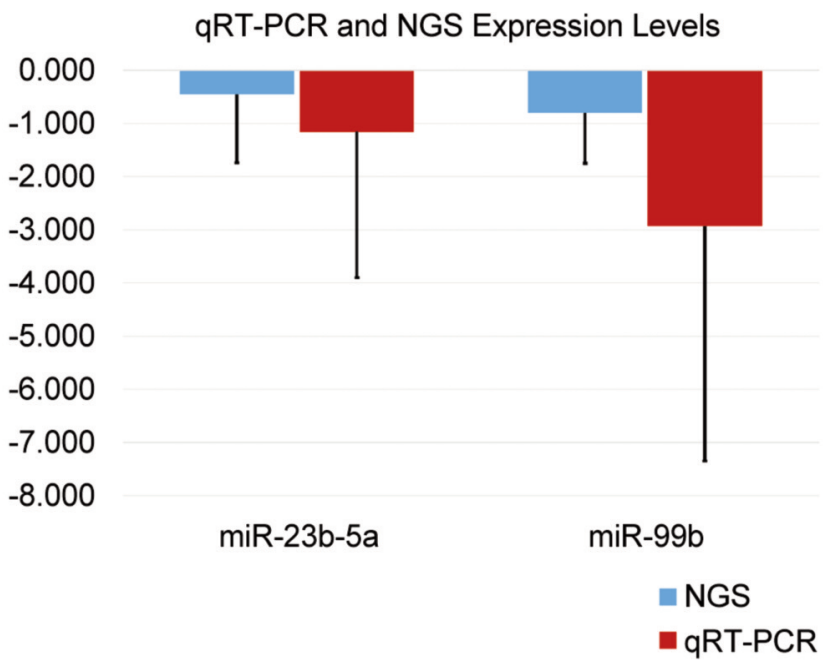

Figure 4. Comparative diagram between $q R T-P C R$ miRNA expression levels and miRNA deep sequencing data.

tyrosine kinase inhibitor resistance (has $01521, p=0.0065$ ) and Central carbon metabolism in cancer (has 05230, $p=0.0045$ ).

Verification of miRNAs expression levels using $q R T-P C R$. Consistent with the sequencing data, qRT-PCR verified the down-regulation of miR-23b-5p and miR-99b-5p in the first trimester maternal plasma in women at risk for LOPE (Figure 4). Both miRNAs were examined for their performance in differentiating between LOPE and control 

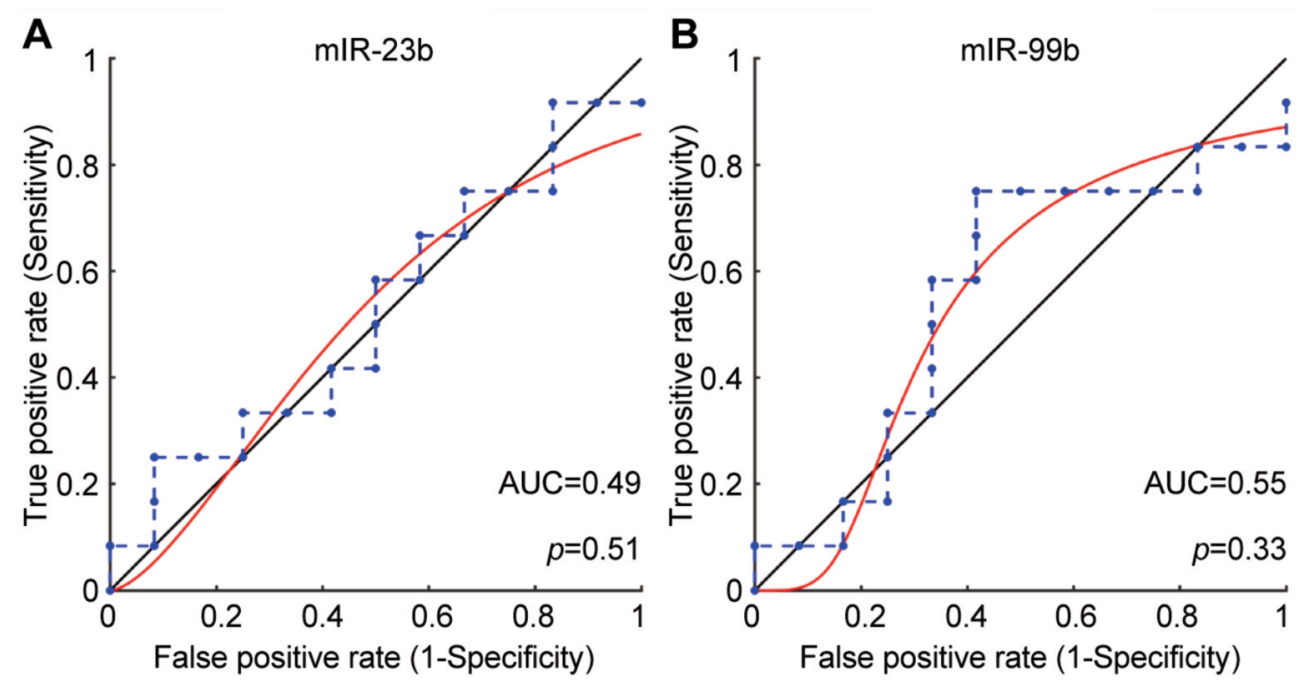

Figure 5. Receiver operating characteristic curves (ROC) of the differentially expressed miRNAs in LOPE as compared to uncomplicated pregnancies. A. $m i R-23 b-5 p$ and, B. $m i R-99 b-5 p$.

samples. The largest AUC was observed for miR-99b-5p $(\mathrm{AUC}=0.55, p=0.33)$ (Figure 5).

\section{Discussion}

In the present study, miRNA deep sequencing in first trimester maternal plasma samples was performed to identify candidate biomarkers associated with the subsequent development of LOPE and provide useful insights regarding pathways associated with the initiation and the development of this pregnancy related complication. In a previous study, we documented differential expression of placental and circulating miRNAs in PE highlighting a significant role of this epigenetic mechanism in the pathogenesis of the complication (18). That study was performed in placenta samples, thus limiting its potential use in prenatal screening. However, circulating miRNAs, which are obtained non-invasively or minimally invasively, represent a source of novel biomarkers that may improve current prenatal screening for PE. Ideally, biomarkers should be able to foresee the disease as early as possible, in the late first trimester when screening for fetal aneuploidies is also performed, to ensure adequate monitoring of women at risk, facilitate the development of preventive strategies and reduce the prevalence and severity of the disease.

Presently, to the best of our knowledge, there are comparatively limited reports focusing on identifying miRNA signatures related to PE in maternal plasma early during pregnancy using deep sequencing, which allows direct miRNA quantification and possesses high sensitivity. In a recent study by Timofeeva et al. (2018), miR-423-5 was identified as a candidate biomarker for the early diagnosis of EOPE (19). Yoffe et al. (2018) also investigated small non-coding RNAs
(ncRNAs), including miRNAs, in the first trimester maternal plasma of $75 \mathrm{PE}$ and control samples and identified 25 transcripts aberrantly expressed between the two groups (20). These transcripts were then used to design a model for the early prediction of PE with an average AUC value of 0.86 .

In the present study, there was significant down-regulation of miR-99b-5p and miR-23b-5p in first trimester maternal plasma in women who later developed LOPE as compared to uncomplicated pregnancies, suggesting the possible involvement of these miRNAs in the pathogenesis of LOPE. MiR-99b-5p is implicated in cell survival, proliferation, cell stress, DNA damage and confers cardioprotection $(21,22)$. Amara et al. (2019), administered Deoxycorticosterone Acetate and $1 \% \mathrm{NaCl}$ in uni-nephrectomized rats and induced hypertension, cardiovascular and renal damage characterized by decreased expression levels of sarco/endoplasmic reticulum $\mathrm{Ca}^{2+}$-ATPase (SERCA2), phosphate-AMP-activated protein kinase (p-AMPK), miR146a and miR-99b-5p and increased levels of miR-155 (22). Interestingly, administration of high dose metformin, an AMPK activator, improved cardiovascular and renal function and restored the expression levels of miR-99b. Of note, miR$99 \mathrm{~b}-5 \mathrm{p}$ was again among the ncRNAs found differentially expressed in maternal plasma samples of first trimester PE pregnancies compared with uncomplicated ones (20).

To the best of our knowledge, there are no reports describing the role of miR-23b-5p in LOPE. miR-23b-5p is a member of the miR-23/27/24 cluster, encoded by a long non-coding RNA transcript referred to as Chromosome 9 Open Reading Frame 3 (C9orf3). Mir-23b-5p regulates target genes related to cell cycle control, proliferation, differentiation and neovascularization (23-27). By targeting 
$T A B 2, T A B 3$ and $I K K$-alpha, miR23b-5p also controls the production of cytokines and growth factors that may lead to vascular damage (28). Additionally, Zhou et al. (2009, 2010 and 2018) demonstrated that it is implicated in angiogenesis (29-32). In PE, unbalanced differentiation between TH17 subtypes has been reported as a result of expansion of TH17 and TH1 cell populations and decreased regulatory T-cell immunity. Nevertheless, no direct link between miR-23b-5p expression levels and the pathogenesis of this pregnancy related complication has yet been documented.

KEGG pathway enrichment analysis revealed that putative targets of the differentially expressed miRNAs have a considerable impact in signaling pathways known to be associated with PE including immune response and insulin resistance. Insulin resistance is an essential pathogenic factor for both PE and gestational diabetes mellitus. In women, who develop preeclampsia increase in maternal insulin resistance is apparent from the first trimester of pregnancy and precedes the clinical onset of the disease (33). According to Founds et al. (2011), insulin resistance positively correlates with inflammation (34). Furthermore, insulin resistance and endothelial dysfunction represent early events in individuals at high risk of developing cardiovascular disease later in life (35). The Epidermal Growth Factor Receptor (EGFR) signaling, which is related to fetal growth restriction, gestational trophoblastic diseases and PE was found significantly enriched in the present study (36). Hastie et al. (2019) showed simultaneous targeting of both EGFR signaling and mitochondrial function using a combination of esomeprazole and metformin results in additive reductions in sFlt-1 production suggesting their use as preventive therapeutic interventions for PE (36).

Conclusively, our findings provided significant insights into the role of two miRNAs that could serve as biomarkers for LOPE. Specifically, following deep sequencing for miRNA plasma profiling, there was good evidence to suggest that miR-23b-5p and miR-99b-5p might be putative prognostic biomarkers for prenatal screening and might also guide the development of novel therapeutic interventions. Yet, further studies using larger heterogeneous cohorts are required to validate the data obtained and establish the performance of both miRNAs in predicting LOPE.

\section{Conflicts of Interest}

The Authors have no conflicts of interest to declare in relation to this study.

\section{Authors' Contributions}

DM and AL: involved in data collection, laboratory analysis and manuscript preparation; GL: performed all data analyses, participated in interpretation of data and in drafting the manuscript; GKP, SK and NV: involved in sample collection, data collection and sample selection; NP: participated in interpretation of data and in editing the manuscript, AK: conceived and designed the study, evaluated and interpreted data, drafted the manuscript and participated in the coordination and supervision of the study. All Authors approved the final manuscript.

Availability of data and material: The datasets used and/or analyzed in the current study are submitted at NCBI (https://www.ncbi. nlm.nih.gov/geo/info/linking.html) with accession no: GSE119799. Other raw data are available upon reasonable request.

\section{Acknowledgements}

DM is a postgraduate student at the MSc "High Risk Pregnancy" at the National and Kapodistrian University of Athens, School of Medicine.

\section{References}

1 Sibai B, Dekker G and Kupferminc M: Pre-eclampsia. Lancet 365(9461): 785-799, 2005. PMID: 15733721. DOI 10.1016/ S0140-6736(05)17987-2

2 American College of Obstetricians and Gynecologists, Task Force on Hypertension in pregnancy: Report of the American College of Obstetricians and Gynecologists' Task Force on Hypertension in Pregnancy. Obstet Gynecol 122(5): 1122-1131, 2013. PMID: 24150027. DOI: 10.1097/01. AOG.0000437382.03963.88

3 Kintiraki E, Papakatsika S, Kotronis G, Goulis DG and Kotsis V: Pregnancy-induced hypertension. Hormones 14(2): 211-223, 2015. PMID: 26158653. DOI: 10.14310/horm.2002.1582

4 Lisonkova S, Sabr Y, Mayer C, Young C, Skoll A and Joseph KS: Maternal morbidity associated with early-onset and lateonset preeclampsia. Obstet Gynecol 124(4): 771-781, 2014. PMID: 25198279. DOI 10.1097/AOG.0000000000000472

5 Myatt L and Roberts JM: Preeclampsia: Syndrome or disease? Curr Hypertens Rep 17(11): 83, 2015. PMID: 26362531. DOI: $10.1007 / \mathrm{s} 11906-015-0595-4$

6 Nicolaides KH: Aspirin versus placebo in pregnancies at high risk for preterm preeclampsia. NEJM 377(24): 2400, 2017. PMID: 28657417. DOI: 10.1056/NEJMoa1704559

7 O'Gorman N, Wright D, Rolnik DL, Nicolaides KH and Poon LC: Study protocol for the randomized controlled trial: combined multimarker screening and randomised patient treatment with ASpirin for evidence-based PREeclampsia prevention (ASPRE). BMJ Open 6(6): e011801, 2016. PMID: 27354081. DOI: 10.1136/bmjopen-2016-011801

8 Anfossi S, Fu X, Nagvekar R and Calin GA: MicroRNAs, regulatory messengers inside and outside cancer cells. Adv Exp Med Biol 1056: 87-108. PMID: 29754176. DOI: 10.1136/ bmjopen-2016-011801

9 Ors-Kumoglu G, Gulce-Iz S and Biray-Avci C: Therapeutic microRNAs in human cancer. Cytotechnology 71(1): 411-425, 2019. PMID: 30600466. DOI: 10.1007/s10616-018-0291-8

$10 \mathrm{Li} \mathrm{Y,} \mathrm{Fang} \mathrm{Y,} \mathrm{Liu} \mathrm{Y} \mathrm{and} \mathrm{Yang} \mathrm{X:} \mathrm{MicroRNAs} \mathrm{in} \mathrm{ovarian}$ function and disorders. J Ovarian Res 8: 51, 2015. PMID: 26232057. DOI: $10.1186 / \mathrm{s} 13048-015-0162-2$

11 Tufekci KU, Meuwissen RL and Genc S: The role of microRNAs in biological processes. Methods Mol Biol 1107: 1531, 2014. PMID: 24272429. DOI: 10.1007/978-1-62703-748-8_2 
12 Gantier MP, McCoy CE, Rusinova I, Saulep D, Wang D, Xu D, Irving AT, Behlke MA, Hertzog PJ, Mackay F and Williams BRG: Analysis of microRNA turnover in mammalian cells following Dicer1 ablation. Nucleic Acids Res 39(13): 56925703, 2019. PMID: 21447562. DOI: $10.1093 / \mathrm{nar} / \mathrm{gkr} 148$

$13 \mathrm{Ha} \mathrm{M}$ and Kim VN: Regulation of microRNA biogenesis. Nat Rev Mol Cell Biol 15(8): 509-524, 2014. PMID: 25027649. DOI: $10.1038 / \mathrm{nrm} 3838$

14 Ohuma EO, Papageorghiou AT, Villar J and Altman DG: Estimation of gestational age in early pregnancy from crown-rump length when gestational age range is truncated: the case study of the INTERGROWTH-21st Project. BMC Med Res Methodol 13: 151, 2013. PMID: 24314232. DOI: 10.1186/1471-2288-13-151

15 ACOG Committee on Obstetric Practice: ACOG practice bulletin: Diagnosis and management of preeclampsia and eclampsia. Number 33, January 2002. Obstet Gynecol 99(1): 159-167, 2002. PMID: 16175681. DOI: 10.1016/s00297844(01)01747-1

16 Bullard JH, Purdom E, Hansen KD and Dudoit S: Evaluation of statistical methods for normalization and differential expression in mRNA-Seq experiments. BMC Bioinformatics 11, 94, 2010. PMID: 20167110. DOI: 10.1186/1471-2105-11-94

17 Zhang B, Kirov S and Snoddy J: WebGestalt: an integrated system for exploring gene sets in various biological contexts. Nucleic Acids Res 33(Web Server issue): W741-748, 2005. PMID: 15980575. DOI: 10.1093/nar/gki475

18 Lykoudi A, Kolialexi A, Lambrou GI, Braoudaki M, Siristatidis C, Papaioanou GK, Tzetis M, Mavrou A and Papantoniou N: Dysregulated placental microRNAs in early and late onset preeclampsia. Placenta 61: 24-32, 2018. PMID: 29277268. DOI: $10.1016 /$ j.placenta.2017.11.005

19 Timofeeva AV, Gusar VA, Kan NE, Prozorovskaya KN, Karapetyan AO, Bayev OR, Chagovets VV, Kliver SF, Iakovishina DY, Frankevich VE and Sukhikh GT: Identification of potential early biomarkers of preeclampsia. Placenta 61: 6171, 2018. PMID: 29277273. DOI: 10.1016/j.placenta.2017.11.011

20 Yoffe L, Gilam A, Yaron O, Polsky A, Farberov L, Syngelaki A, Nicolaides K, Hod M and Shomron N: Early detection of preeclampsia using circulating small non-coding RNA. Sci Rep 8(1): 3401, 2018. PMID: 29467498. DOI: 10.1038/s41598-018-21604-6

21 Ye X, Luo H, Chen Y, Wu Q, Xiong Y, Zhu J, Diao Y, Wu Z, Miao $J$ and Wan J: MicroRNAs 99b-5p/100-5p regulated by endoplasmic reticulum stress are involved in abeta-induced pathologies. Front Cell Neurosci 7: 210, 2015. PMID: 26635599. DOI: $10.3389 /$ fnagi.2015.00210

22 Amara VR, Surapaneni SK and Tikoo K: Metformin attenuates cardiovascular and renal injury in uninephrectomized rats on DOCA-salt: Involvement of AMPK and miRNAs in cardioprotection. Toxicol Appl Pharm 362: 95-104, 2019. PMID: 30290168. DOI: 10.1016/j.taap.2018.10.004

23 Chhabra R, Dubey R and Saini N: Cooperative and individualistic functions of the microRNAs in the miR-23a 27a 24-2 cluster and its implication in human diseases. Mol Cancer 9: 232, 2010. PMID: 20815877. DOI: 10.1186/1476-4598-9-232

24 Lee J, Li Z, Brower-Sinning R and John B: Regulatory circuit of human microRNA biogenesis. PLoS Comput Biol 3(4): e67, 2007. PMID: 17447837. DOI: 10.1371/journal.pcbi.0030067

25 O'Connell RM, Kahn D, Gibson WS, Round JL, Scholz RL, Chaudhuri AA, Kahn ME, Rao DS and Baltimore D: MicroRNA155 promotes autoimmune inflammation by enhancing inflammatory
T cell development. Immunity 33(4): 607-619, 2010. PMID: 20888269. DOI: 10.1016/j.immuni.2010.09.009

26 Pan SJ, Zhan SK, Pei BG, Sun QF, Bian LG and Sun BM: MicroRNA-149 inhibits proliferation and invasion of glioma cells via blockade of AKT1 signaling. Int J Immunopathol Pharmacol 25(4): 871-881, 2012. PMID: 23298478. DOI: $10.1177 / 039463201202500405$

27 Zhu S, Pan W, Song X, Liu Y, Shao X, Tang Y, Liang D, He D, Wang H, Liu W, Shi Y, Harley JB, Shen N and Qian Y: The microRNA miR-23b suppresses IL-17-associated autoimmune inflammation by targeting TAB2, TAB3 and IKK-alpha. Nat Med 18(7): 1077-1086, 2012. PMID: 23298478. DOI: 10.1177/ 039463201202500405

28 Morton LT, Situnayake D and Wallace GR: Genetics of Behcet's disease. Curr Opin Rheumatol 28(1): 39-44, 2016. PMID: 26599381. DOI: 10.1097/BOR.0000000000000234

29 Qi P, Xu MD, Shen XH, Ni SJ, Huang D, Tan C, Weng WW, Sheng WQ, Zhou XY and Xiang Du: Reciprocal repression between TUSC7 and miR-23b in gastric cancer. Int J Cancer 137(6): 1269-1278, 2015. PMID: 25765901. DOI: 10.1002/ ijc. 29516

30 Zhou B, Yuan W and Li X: LncRNA Gm5091 alleviates alcoholic hepatic fibrosis by sponging miR- 27b/23b/24 in mice. Cell Biol Int 42(10): 1330-1339, 2018. PMID: 29935035. DOI: 10.1002/cbin.11021

31 Zhou R, Hu G, Gong AY and Chen XM: Binding of NF-kappaB p65 subunit to the promoter elements is involved in LPS-induced transactivation of miRNA genes in human biliary epithelial cells. Nucleic Acids Res 38(10): 3222-3232, 2010. PMID: 20144951. DOI: $10.1093 / \mathrm{nar} / \mathrm{gkq056}$

32 Zhou R, Hu G, Liu J, Gong AY, Drescher KM and Chen XM: NF-kappaB p65-dependent transactivation of miRNA genes following Cryptosporidium parvum infection stimulates epithelial cell immune responses. PLoS Pathog 5(12): e1000681, 2009. PMID: 19997496. DOI: 10.1371/journal.ppat.1000681

33 Akolekar R, Syngelaki A, Poon L, Wright D and Nicolaides KH: Competing risks model in early screening for preeclampsia by biophysical and biochemical markers. Fetal Diagn Ther 33(1): 8-15, 2013. PMID: 22906914. DOI: $10.1159 / 000341264$

34 Founds SA, Catov JM, Gallaher MJ, Harger GF, Markovic N and Roberts JM: Is there evidence of separate inflammatory or metabolic forms of preeclampsia? Hypertens Pregnancy 30(1): 110, 2011. PMID: 20462373. DOI: 10.3109/10641950903322907

$35 \mathrm{Wu}$ Y, Xiong X, Fraser WD and Luo ZC: Association of uric acid with progression to preeclampsia and development of adverse conditions in gestational hypertensive pregnancies. Am J Hypertens 25(6): 711-717, 2012. PMID: 22378034. DOI: 10.1038/ajh.2012.18

36 Hastie R, Brownfoot FC, Pritchard N, Hannan NJ, Cannon P, Nguyen V, Palmer K, Beard S, Tong S and Kaitu'u-Lino TJ: EGFR (Epidermal Growth Factor Receptor) signaling and the mitochondria regulate sFlt-1 (Soluble FMS-Like Tyrosine Kinase-1) secretion. Hypertension 73(3): 659-670, 2019. PMID: 30636550. DOI: 10.1161/HYPERTENSION AHA.118.12300

Received May 10, 2020

Revised May 28, 2020

Accepted May 29, 2020 